\title{
Die Reform der Komitologie mit und nach dem Vertrag von Lissabon: The End Of The World As We Know It?
}

\author{
Annette Elisabeth Töller*
}

In der Europäischen Union hat sich in den vergangenen Jahrzehnten eine komplexe Verwaltungsstruktur herausgebildet, deren wichtigste Elemente die Europäische Kommission, die Komitologie, die wachsende Anzahl von Agenturen und die Verwaltungsnetzwerke sind. ${ }^{1}$ Komitologie bezeichnet die Delegation von Durchführungsbefugnissen an die Kommission und deren Wahrnehmung unter formalisierter Mitarbeit von Ausschüssen aus mitgliedstaatlichen Delegierten. 2011 waren es 268 Ausschüsse (davon die meisten in den Bereichen Umwelt, Unternehmen und Industrie sowie Verkehr und Energie), die in 783 Sitzungen an Beschlüssen von 1.625 Durchführungsmaßnahmen beteiligt waren. ${ }^{2}$ Während ständige Veränderung eines der wichtigsten Charakteristika dieser Verwaltungsstrukturen (wie auch der sonstigen europäischen Institutionenordnung) darstellt, ${ }^{3}$ war die Komitologie als europäische Verwaltungsstruktur mit dem und infolge des Lissaboner Vertrag(-s) einer Reform unterworfen, wie sie in dieser Radikalität nur selten anzutreffen ist. Erstens wurde mit den „delegierten Rechtsakten“ gemäß Art. 290 Vertrag über die Arbeitsweise der Europäischen Union (AEUV) eine vollkommen neue Kategorie von Rechtsakten mit einem ebenfalls neuen Verfahren eingeführt. Diese werden nicht im Komitologieverfahren, sondern von der Kommission allein unter Revokationsrechten von Parlament und Rat als Gesetzgeber beschlossen. Damit wurde das Anwendungsgebiet der Komitologie potenziell gravierend beschnitten. Zweitens ist im Primärrecht mit Art. 291 AEUV das Entscheidungsverfahren für die Regeln der Komitologie, die bisher (in den Jahren 1987, 1999 und 2006) durch Ratsbeschlüsse festgelegt worden waren, so geändert worden, dass das Parlament nun im ordentlichen Gesetzgebungsverfahren gleichberechtigt beteiligt ist. Drittens wurden in der so beschlossenen Komitologieverordnung von 2011 die Komitologieverfahren vereinfacht, wobei an die Stelle des Rekurses zum Rat ein Berufungsausschuss tritt.

Der folgende Beitrag verfolgt das Ziel, diese Reform vor dem Hintergrund der bisherigen Konfiguration der Komitologie zum einen zu erläutern und zum anderen die Ursachen für diesen radikalen Wandel herauszuarbeiten. Seine voraussichtlichen Effekte können nur in Ansätzen skizziert werden, da hierzu bislang keine systematischen empirischen Erkenntnisse vorliegen. Die wissenschaftliche Literatur hat sich zwar in den vergangenen Jahren zuneh-

* Prof. Dr. Annette Elisabeth Töller ist Professorin für Politikwissenschaft mit dem Schwerpunkt Politikfeldanalyse an der FernUniversität in Hagen.

1 Thurid Hustedt/Arndt Wonka/Michael Blauberger/Annette Elisabeth Töller/Renate Reiter: Verwaltungsstrukturen in der Europäischen Union. Kommission, Komitologie, Agenturen und Verwaltungsnetzwerke, Wiesbaden 2013.

2 Europäische Kommission: Bericht der Kommission über die Tätigkeit der Ausschüsse im Jahr 2011, KOM (2012) 684, hier S. 7-13.

3 Vgl. Helen Wallace: An institutional anatomy and five policy modes, in: Helen Wallace/Mark A. Pollack/Alasdair R. Young (Hrsg.): Policy-Making in the European Union, New York 2010, S. 69-104, hier. S. 4-5; Hustedt et al.: Verwaltungsstrukturen in der Europäischen Union, 2013, hier S. 23-25. 
mend mit der Reform befasst, ${ }^{4}$ sie weist dabei aber erstaunliche Blindstellen auf. Erstens betrachten die meisten Autoren ${ }^{5}$ die Reform - explizit oder implizit - als originäres Resultat des 2009 in Kraft getretenen Lissaboner Vertrags und vernachlässigen dabei, dass diese Reform nahezu identisch bereits Inhalt des aus der Arbeit des Konvents zur Zukunft der Europäischen Union (Konvent) hervorgegangenen Entwurfs für den Vertrag über eine Verfassung für Europa (Verfassungsvertrag) von 2003 war. ${ }^{6}$ Zweitens wird einige Energie darauf verwendet, das Resultat der 2011 in Kraft getretenen Komitologieverordnung zu erklären, ${ }^{7}$ während das Zustandekommen der sehr viel gravierenderen Veränderungen im Primärrecht, ${ }^{8}$ das intuitiv viel mehr Überraschung hervorrufen sollte, bislang kaum Gegenstand von Erklärungsversuchen gewesen ist. ${ }^{9}$ Und drittens sind Erklärungsversuche, die ganz überwiegend die theoretische Perspektive des Rational-Choice-Institutionalismus anwenden, letztlich nicht in der Lage, die hier vorgefundenen Veränderungen zu erklären. Deutlich weiter kommt man, wenn man auch den Historischen und den Soziologischen Institutionalismus zur Erklärung heranzieht (siehe unten). Der vorliegende Beitrag erhebt nicht den Anspruch, eine systematische theoriegeleitete empirische Analyse zu leisten. Vielmehr handelt es sich um eine theoretisch informierte Gesamtbetrachtung. Der Beitrag ist folgendermaßen aufgebaut: Zunächst wird die ,alte Komitologie' bis 1999 kurz vorgestellt, denn es lässt sich die Relevanz der Komitologie für das Gesamtsystem der Europäischen Union nur verstehen und der Wandel lässt sich nur betrachten, wenn der Status quo ante bekannt ist. Dann wird die längere Reformphase im neuen Jahrtausend betrachtet, die, so das Argument, zwar recht verschlungen verlaufen ist, aber die Reformen mit und nach dem Lissaboner Vertrag wesentlich vorbereitet hat. Schließlich wird die im Lissaboner Vertrag enthaltene Reform genauer betrachtet, um in einem letzten Schritt nach Ursachen für diesen gravierenden Wandel zu suchen. Der Beitrag schließt mit einem Resümee.

\section{Die ,alte' Komitologie bis 1999 - Grundlagen und Konflikte}

Die Komitologie als europäische Verwaltungsstruktur wurde nicht am Reißbrett entworfen. Sie entstand vielmehr zunächst dezentral und in gewissem Maße inkrementell in ein-

4 Vgl. zum Beispiel Herwig C. Hofmann: Legislation, Delegation and Implementation under the Treaty of Lisbon: Typology Meets Reality, in: European Law Journal 4/2009, S. 482-505; Dominique Ritleng: La délégation du pouvoir legislatif de l'Union Européenne, in: Chemins d'Europe. Mélanges en l'honneur de Jean Paul Jacqué, Paris 2010, S. 559-576; Paul Craig: Delegated Acts, Implementing Acts and the New Comitology Regulation, in: European Law Review 5/2011, S. 671-687; Thomas Christiansen/Mathias Dobbels: Comitology and delegated acts after Lisbon: How the European Parliament lost the implementation game, in: European Integration online Papers 13/2012; Thomas Christiansen/Mathias Dobbels: Non-Legislative Rule Making after the Lisbon Treaty: Implementing the New System of Comitology and Delegated Acts, in: European Law Journal 1/2013, S. 42-56; Gijs J. Brandsma/Jens Blom-Hansen: Negotiating the Post-Lisbon Comitology System: Institutional Battles Over Delegated Decision-Making, in: Journal of Common Market Studies 6/2012, S. 939-957; Adrienne Héritier/ Catherine Moury/Carina Bischoff/Carl Fredrik Bergström: Changing Rules of Delegation. A Contest for Power in Comitology, Oxford 2013.

5 Vgl. zum Beispiel Brandsma/Blom-Hansen: Negotiating the Post-Lisbon Comitology System, 2012, S. 939; Héritier et al.: Changing Roles of Delegation, 2013, S. 46.

6 Diese falsche Wahrnehmung kritisiert unter anderem Antoine Buchet von der Kommission, in: Antoine Buchet/ Katrin Huber/Adrienne Héritier/Catherine Moury/Carina Bischoff/Carl-Fredrik Bergström: Changing Rules of Delegation, Florenz, 20. Januar 2012, Min. 31:30, abrufbar unter: www.youtube.com/watch?v=3MTF08hiZhA \&list=UUtt-BiJPPfepbUMR1CzL2Tg\&feature=player_detailpage (letzter Zugriff: 25.7.2013).

7 Christiansen/Dobbels: Comitology and delegated acts after Lisbon, 2012; Brandsma/Blom-Hansen: Negotiating the Post-Lisbon Comitology System, 2012.

8 Art. 290 und Art. 291 AEUV.

9 Vgl. aber Jonas Bering Liisberg: The EU Constitutional Treaty and its distinction between legislative and nonlegislative acts - Oranges into apples?, New York University School of Law: Jean Monnet Working Paper 01/06; am Rande Héritier et al: Changing Roles of Delegation, 2013, S. 41-42. 
zelnen Politikfeldern, breitete sich dann langsam aus und differenzierte sich. Ihren Beginn nahm sie in der Agrarpolitik der frühen 1960er Jahre, als die Notwendigkeit entstand, die neuen Agrarmarktordnungen zu verwalten. ${ }^{10}$ Während die Kommission zunächst nur beratende Ausschüsse vorgeschlagen hatte, denen die Mitgliedstaaten eher ablehnend gegenüberstanden, einigte man sich schließlich auf das von Walter Hallstein vorgeschlagene Verwaltungsausschussverfahren, in dem die Kommission zunächst Beschlüsse fassen und diese auch durchführen konnte. ${ }^{11}$ Nur wenn der Ausschuss mit einer qualifizierten Mehrheit gegen den Beschluss stimmte, würde der Rat befasst, der eine anderslautende Entscheidung treffen konnte. ${ }^{12}$ Das Verfahren wurde 1962 für eine ganze Reihe von Agrarmarktordnungen mit insgesamt zehn Ausschüssen eingerichtet. Schon damals protestierte das Europäische Parlament. ${ }^{13}$ Damit war ein Prototyp geschaffen, der die Frage der Kontrolle durch die Mitgliedstaaten in gewisser Weise institutionalisierte und damit für alle Beteiligten Komplexität und Unsicherheit reduzierte. ${ }^{14}$ Entlang dieses Pfades entwickelte sich die Komitologie weiter. Im Rahmen der Ausweitung der Entwicklungshilfe für Afrika wurde 1963 das erste Regelungsausschussverfahren eingeführt. ${ }^{15}$ Hier konnte die Kommission eine Maßnahme nur beschließen, wenn sie eine qualifizierte Mehrheit im Ausschuss für diese Maßnahmen erhielt (andernfalls musste der Rat befasst werden - der sogenannte Rekurs). Das Regelungsausschussverfahren wurde 1968 auch im Kontext der anstehenden Gestaltung der Durchführungskompetenzen für die Zollunion eingeführt. Allerdings kam hier ein weiteres Verfahrenselement hinzu, das später als ,Filet' (Sicherheitsnetz) bekannt wurde: Fasste der Rat (auf Vorschlag der Kommission) im Falle eines Rekurses nicht binnen drei Monaten einen Beschluss, konnte die Kommission die Maßnahme dennoch erlassen. ${ }^{16}$ Im offenbar sensiblen Bereich des Futtermittel- und Veterinärrechts wurde schließlich das ,Contrefilet ${ }^{6}$ eingeführt: der Rat sollte eine Beschlussfassung der Kommission (Filet, siehe oben) mit einfacher Mehrheit verhindern können. 1970 wurde der Gerichtshof der Europäischen Gemeinschaft $(\mathrm{EuGH})$ erstmals mit dem Phänomen befasst, an dem er im Wesentlichen nichts auszusetzen hatte, da die Ausschüsse nicht anstelle der Kommission entschieden. ${ }^{17}$ Im Laufe der 1970er und frühen 1980er Jahre, die man als Konsolidierungsphase der Komitologie bezeichnen kann, wurden immer neue Ausschüsse eingesetzt. Neben einer Zunahme der Ausschüsse kam es zu einem Wildwuchs der Verfahrensvarianten.

War also die Verwendung der Komitologie bis Mitte der 1980er Jahre einer eher inkrementellen Logik gefolgt, so kam es mit der Einheitlichen Europäischen Akte 1987 erstmals zu einem bewussten und jenseits der einzelnen Politikfelder geplanten Einsatz dieser Verwaltungsstruktur. Im Kontext des neu in Angriff genommenen Binnenmarktprojekts sollte die Komitologie (neben der tatsächlichen Anwendung der qualifizierten Mehrheit im Rat und dem Prinzip der gegenseitigen Anerkennung) eine Strategie darstellen, um - vor allem angesichts des erwartbar steigenden Regulierungsbedarfs - die Entscheidungsfähigkeit der

10 Helmut Schmitt von Sydow: Organe der erweiterten Europäischen Gemeinschaften - die Kommission, BadenBaden 1980, S. 139.

11 Jens Blom-Hansen: The origins of the EU comitology system: a case of informal agenda-setting by the Commission, in: Journal of European Public Policy 2/2008, S. 208-226, hier S. 217.

12 Schmitt von Sydow: Organe der erweiterten Europäischen Gemeinschaften, 1980, S. 140.

13 Blom-Hansen: The origins of the EU comitology system, 2008, S. 217.

14 Annette Elisabeth Töller: Komitologie. Theoretische Bedeutung und praktische Arbeitsweise von Durchführungsausschüssen in der Europäischen Union am Beispiel der Umweltpolitik, Opladen 2002, S. 231-237; BlomHansen: The origins of the EU comitology system, 2008, S. 223.

15 Ebenda, S. 219.

16 Töller: Komitologie, 2002, hier S. 235; Blom-Hansen: The origins of the EU comitology system, 2008, S. 222.

17 EuGH, Rs. C-25/70 (Einfuhr- und Vorratsstelle für Getreide und Futtermittel/Köster u. a.), Slg. 1970, S. 1162. 
Gemeinschaft zu verbessern. ${ }^{18}$ In diesem Zusammenhang wurden die Grundlagen der Komitologie erstmals in Art. 145 des geänderten Vertrags zur Gründung der Europäischen Gemeinschaft (EGV) festgelegt, wo es hieß:

„Zur Verwirklichung der Ziele und nach Maßgabe dieses Vertrags [...] überträgt der Rat der Kommission in den von ihm angenommenen Rechtsakten die Befugnisse zur Durchführung der Vorschriften, die er erlässt. Der Rat kann bestimmte Modalitäten für die Ausübung dieser Befugnisse festlegen. [...] Die oben genannten Modalitäten müssen den Grundsätzen und Regeln entsprechen, die der Rat auf Vorschlag der Kommission nach Stellungnahme des Europäischen Parlaments vorher einstimmig festgelegt hat.“

Diese „Grundsätze und Regeln“ wurden durch den sogenannten (ersten) Komitologiebeschluss von $1987^{19}$ festgelegt. Art. 145 EGV wurde durch den Vertrag von Amsterdam unverändert zu Art. 202 EGV.

Die Grundkonstellation nach Art. 202 EGV bestand darin, dass der Kommission mit einem Rechtsakt bestimmte, mehr oder weniger genau definierte Durchführungsbefugnisse übertragen wurden. Diese Befugnisse zum Beschluss bestimmter Maßnahmen wurden dann unter Anwendung unterschiedlicher Verfahren zur Beteiligung eines Ausschusses aus Delegierten der Mitgliedstaaten wahrgenommen. Die Befugnisse selber konnten sehr unterschiedlicher Natur sein. Oft ging es um die Operationalisierung von Richtlinien, indem zum Beispiel in Anhängen bestimmte Produktgruppen definiert wurden, für die bestimmte Regelungen gelten, indem bestimmte Messtechniken festgelegt oder Verfahren konkretisiert oder auch angepasst wurden. Zudem erfordern einige Rechtsakte auch das Treffen von administrativen Einzelfallentscheidungen (zum Beispiel über die Zulassung bestimmter Stoffe oder Produkte, die Festlegung von Preisen und Quoten oder die Verteilung von Mitteln), an denen alle Mitgliedstaaten beteiligt sein müssen. ${ }^{20}$ Diese Entscheidungen sind in der Regel technischer Natur, können aber mitunter auch sehr sensible politische Fragen aufwerfen.

Über das Treffen formeller Beschlüsse hinaus haben die Komitologieausschüsse aber weitere Funktionen (streng rechtlich gesehen operieren dann dieselben Delegierten als Expertenausschüsse): So arbeitet man hier an der gemeinsamen Auslegung zentraler Begriffe und Kategorien und damit an einem gemeinsamen Verständnis einer Regelung. Überdies wäre die Kommission ohne die Informationen aus den Mitgliedstaaten sowie auch die politikfeldspezifische Expertise der nationalen Delegierten kaum in der Lage, ein Programm vernünftig umzusetzen. ${ }^{21}$ Schließlich bedürfen auch solche auf den ersten Blick technischen Regelungen der Zustimmung aus den Mitgliedstaaten, um dann auch eine reibungslose Um-

18 Töller: Komitologie, 2002, S. 144.

19 Vgl. Erklärung zu den Durchführungsbefugnissen der Kommission, in: Amtsblatt der EG, Nr. L 169 vom 19. Juni 1987, S. 24. Eigentlich waren dies nur Regeln, keine Grundsätze; vgl.: Herwig C. Hofmann/Annette E. Töller: Zur Reform der Komitologie - Regeln und Grundsätze für die Verwaltungskooperation im Ausschusssystem der Europäischen Gemeinschaft, in: Staatswissenschaften und Staatspraxis 2/1998, S. 209-239.

20 Zum Beispiel Töller: Komitologie, 2002, hier S. 351; Herwig C. Hofmann: Legislation, Delegation and Implementation under the Treaty of Lisbon: Typology Meets Reality, in: European Law Journal 4/2009, S. 482-505, hier S. 495.

21 Vgl. Töller: Komitologie, 2002; Martijn Groenleer: Regulatory Governance in the European Union: The Political Struggle Over Committees, Agencies and Networks, in: David Levi-Faur (Hrsg.): Handbook on the Politics of Regulation, 2011, S. 548-560; Hustedt et al.: Verwaltungsstrukturen in der Europäischen Union, 2013. 
setzung zu gewährleisten. Die Komitologie ermöglicht also auch die Generierung und Aufrechterhaltung von Folgebereitschaft in den Mitgliedstaaten für das jeweilige Programm. ${ }^{22}$

\section{Interinstitutionelle Konflikte}

Allerdings war die Komitologie seit jeher Gegenstand interinstitutioneller Konflikte. Bereits im Vorfeld des Komitologiebeschlusses von 1987 hatte sich der Widerstand des Europäischen Parlaments geregt, das über die Komitologie umfassend unterrichtet, ${ }^{23}$ im Verwaltungsausschussverfahren im Rekursfall durch Anhörung beteiligt werden und die Regelungsausschussverfahren ganz abgeschafft sehen wollte. ${ }^{24}$ Der Rat ging aber damals auf keine der Forderungen des Parlaments ein. ${ }^{25}$ Damit begann ein gleichermaßen heftiger wie auch komplexer interinstitutioneller Konflikt, bei dessen Beschreibung in der Literatur auch mit Metaphern des Krieges nicht gespart wurde. ${ }^{26}$ Hier stand einmal die Kommission gegen den Rat, das Parlament gegen den Rat und schließlich auch noch das Parlament gegen die Kommission. Dabei war die Kommission gar nicht gegen die Komitologie als solche, wehrte sich aber gegen das Contrefilet-Verfahren, gegen den Einsatz von Komitologieverfahren im Bereich ihrer Haushaltskompetenzen sowie gegen die Fälle, in denen sich der Rat die Durchführungsbefugnisse selber vorbehielt. ${ }^{27}$ Das Parlament hingegen lehnte die Komitologie als solche ab und sah die Ausschüsse als trojanische Pferde, durch die die Umsetzung von Gemeinschaftspolitiken unbemerkt renationalisiert wurde. ${ }^{28}$ Zugleich fürchtete das Parlament eine Schwächung der ihm politisch verantwortlichen Kommission.

Ende der 1980er Jahre scheiterte das Europäische Parlament mit dem Versuch, auf dem Klageweg vor dem EuGH gegen die Komitologie vorzugehen. ${ }^{29}$ Nach der Vertragsrevision von ,Maastricht', Anfang der 1990er Jahre, spitzte sich der Konflikt weiter zu. Waren die legislativen Rechte des Europäischen Parlaments mit der Einführung des Kooperationsver-

22 Die politikwissenschaftliche Diskussion über die Komitologie kreiste viele Jahre vor allem um die Frage, ob die Ausschüsse eher als ,Watchdogs' der Mitgliedstaaten oder als technokratische Fachbruderschaften zu verstehen sind, so zum Beispiel Christian Joerges/Jürgen Neyer: Transforming strategic interaction into deliberative problem-solving: European comitology in the foodstuff sector, in: Journal of European Public Policy 4/1997, S. 609-625; Mark A. Pollack: Control Mechanism Or Deliberative Democracy? Two Images of Comitology, in: Comparative Political Studies 1/2003, S. 125-155; Renaud Dehousse: Comitology: who watches the watchmen?, in: Journal of European Public Policy 5/2003, S. 798-813. Empirische Studien haben inzwischen gezeigt, dass sich die Arbeit konkreter Ausschüsse immer als jeweils zu bestimmende Mischung aus beidem darstellt. Vgl. Wolfgang Wessels: Comitology: fusion in action. Politico-administrative trends in the EU system, in: Journal of European Public Policy 2/1998, S. 209-234; Töller: Komitologie, 2002; Sebastian Huster: Europapolitik aus dem Ausschuss - Innenansichten des Ausschusswesens der EU, Wiesbaden 2008; Jens Blom-Hansen/Gijs Brandsma: The EU Comitology System: Intergovernmental Bargaining or Deliberative Supranationalism?, in: Journal of Common Market Studies 4/2009, S. 719-740; Gijs J. Brandsma: Accountable Comitology?, in: Mark Bovens/Deirdre Curtin/Paul 't Hart (Hrsg.): The Real World of EU Accountability. What Deficit?, New York 2010, S. 150-173, hier S. 152-153.

23 Dies hatte das Europäische Parlament bereits 1968 erstmals gefordert.

24 Europäisches Parlament: Entschließung über die Gemeinschaftsverfahren zur Durchführung des abgeleiteten Gemeinschaftsrechts, in: Amtsblatt der EG, Nr. C 108 vom 19. Oktober 1968, S. 37-38, hier S. 38; Entschließung zum Abschluß des Verfahrens der Konsultation des Europäischen Parlaments zu dem Vorschlag der Kommission der Europäischen Gemeinschaften an den Rat für eine Verordnung zur Festlegung der Modalitäten für die Ausübung der Durchführungsbefugnisse, die der Kommission übertragen werden, in: Amtsblatt der EG, Nr. C 297 vom 24. November 1986, S. 94-96, hier S. 94.

25 Töller: Komitologie, 2002, hier S. 240.

26 Vgl. Charles Reich: Le Parlement Européen et la „comitologie“. Revue du Marché Commun 1990, S. 319-323.

27 Töller: Komitologie, 2002, hier S. 244-255.

28 Kieran St. Clair Bradley: The European Parliament and Comitology: On the road to nowhere, in: European Law Journal 3/1997, S. 230-254.

29 EuGH, Rs. $302 / 87$ (Parlament/Rat), Slg. 1988, 5615. 
fahrens bereits gestärkt worden, so empfand sich das Parlament seit der Einführung des Mitentscheidungsverfahrens durch den Maastrichter Vertrag - zu Recht - als Bestandteil des delegierenden Gesetzgebers. Art. 145 EGV und der Komitologiebeschluss hingegen kannten nur den ,Rat' als delegierendes Organ. Aus dieser institutionellen, Schieflage“ entstand Mitte der 1990er Jahre einer der heftigsten interinstitutionellen Konflikte der Union überhaupt. Das erste Gesetzgebungsverfahren, das das Europäische Parlament im Mitentscheidungsverfahren scheitern ließ, scheiterte an der Komitologie. ${ }^{30}$ Das Parlament setzte sowohl seine Rechte in der Gesetzgebung als auch bei der Haushaltsbewilligung ein, um seine Positionen zur Komitologie zu vertreten und Mitte der 1990er Jahre zumindest erste prozedurale Zugeständnisse zu erreichen. Im Konflikt zwischen Parlament und Kommission ging es auch um die Intransparenz des Systems, die es dem Parlament erschwerte, die Komitologie politisch zu kontrollieren. Ebenfalls Mitte der 1990er Jahre gelang es dem Europäischen Parlament, indem es mehrfach Finanzmittel für die Komitologie auf Eis legte, die Kommission zu einem ersten umfassenden Bericht über die Komitologie zu zwingen. ${ }^{31}$

Mit dem Komitologiebeschluss von 1999 wurden zum einen die Verfahren etwas verändert, zum anderen erhielt das Parlament erstmals Informations- und Beteiligungsrechte, die seiner gestärkten Position als Gesetzgeber entsprechen sollten: Das Parlament konnte (in Ausschuss- und Rekursphase) nun darüber wachen, ob die in Komitologieverfahren getroffenen Beschlüsse im Rahmen der delegierten Kompetenzen blieben. Eine mögliche Folge war, dass die Kommission die Entscheidung ins Gesetzgebungsverfahren zurückholte, also die Ebene wechselte. ${ }^{32}$ Außerdem wurde die Kommission verpflichtet, jährliche Berichte über die Arbeit der Ausschüsse vorzulegen. Allerdings waren die Kontrollrechte damit relativ eng definiert. Das Europäische Parlament erhielt nicht das Recht, eine in der Komitologie getroffene Entscheidung zurückzuholen.

\section{Verschlungene Reformpfade im neuen Jahrtausend}

$\mathrm{Zu}$ Beginn des neuen Jahrtausends fand nun eine merkwürdige Gleichzeitigkeit recht unterschiedlicher Entwicklungen statt. Erstens wurde mit dem Lamfalussy-Verfahren zur Regulierung des Finanzdienstleistungssektors die Komitologie als Bestandteil einer neuen, ,besseren' Regulierungsstrategie eingeführt (siehe unten), was weit über das Politikfeld hinaus von Relevanz war. Zweitens tagte zwischen 2002 und 2003 der Europäische Konvent, der auch für die Komitologie sehr weitreichende Veränderungen vorschlug, die letztlich Eingang in das Primärrecht fanden (siehe unten). Da jedoch zwischen Konvent und dem Inkrafttreten des Lissaboner Vertrags hinsichtlich der Komitologie ein institutionelles Vakuum entstand, wurde drittens 2006 ein neuer Ratsbeschluss zu den Komitologieverfahren verabschiedet, der den Vorstellungen des Parlaments zumindest teilweise entgegen kam.

\section{Lamfalussy-Verfahren als Reformimpuls}

Ein wichtiger Reformimpuls, der nicht aus der konstitutionellen Debatte, sondern aus den Regelungsbedürfnissen eines spezifischen Politikfeldes kam, war die Diskussion über das

30 Vgl. Bradley: The European Parliament and Comitology, 1997; Töller: Komitologie, 2002, hier S. 252.

31 Töller: Komitologie, 2002, hier S. 253-254.

32 Beschluss des Rates vom 28. Juni 1999 zur Festlegung der Modalitäten für die Ausübung der der Kommission übertragenen Durchführungsbefugnisse, in: Amtsblatt der EU, Nr. L 184 vom 17. Juli 1999, S. 23-26. Im Einzelnen dazu Koen Lenaerts/Amaryllis Verhoeven: Towards a Legal Framework for Executive Rule-Making in the EU? The Contribution of the New Comitology Decision, in: Common Market Law Review 3/2000, S. 645-686. 
sogenannte Lamfalussy-Verfahren. ${ }^{33}$ Ende der 1990er Jahre erschien der Kommission die Gesetzgebung im Mitentscheidungsverfahren als zu „mühselig und langsam“, um den Binnenmarkt für Finanzdienstleistungen - wie im Aktionsplan der Kommission für Finanzdienstleistungen von 1999 dargelegt - endlich zu realisieren. ${ }^{34}$ Der Rat setzte daraufhin im Jahr 2000 einen ,Ausschuss der Weisen“ unter Vorsitz von Alexandre Lamfalussy ein, der Vorschläge machen sollte, wie dieses Ziel realisiert werden könnte. Der Ausschuss identifizierte neben der Schwerfälligkeit des Mitentscheidungsverfahrens das Problem, dass das bisherige System nicht zwischen wesentlichen Grundsätzen und praktischen Vorschriften für die tägliche Anwendung unterscheide, weshalb Erstere zu detailliert ausfielen und Letztere zu schwer zu aktualisieren seien. ${ }^{35}$ Nachdem die Idee einer europäischen Regulierungsagentur nach amerikanischem Vorbild schnell wieder verworfen worden war, wurde 2002 zunächst für die Regulierung des Wertpapiersektors das sogenannte Lamfalussy-Verfahren entwickelt, das dann 2004 auch auf den Bereich Banken sowie Versicherungen und Pensionsfonds - und damit auf den gesamten Finanzsektor - ausgedehnt und im Weißbuch „Europäisches Regieren“ der Europäischen Kommission aufgegriffen wurde. ${ }^{36}$

Im Zusammenhang mit diesem Verfahren wurden zunächst zwei Ausschüsse geschaffen: der Europäische Wertpapierausschuss aus nationalen Delegierten (ESC - ein Regelungsausschuss gemäß Komitologiebeschluss von 1999) sowie ein beratender Ausschuss der europäischen Wertpapierregulierungsbehörden (CESR). Die Ausschüsse sollten letztlich nicht nur der europäischen Regelsetzung, sondern auch der EU-weiten Vernetzung der nationalen Wertpapierregulierer dienen. Für die anderen beiden Bereiche wurden später analog der europäische Bankenausschuss sowie der europäische Ausschuss für das Versicherungswesen und die betriebliche Altersversorgung als Komitologieausschüsse sowie als beratende Ausschüsse der Ausschuss der europäischen Bankenaufsichtsbehörden (CEBS) und der Ausschuss der europäischen Aufsichtsbehörden für das Versicherungswesen und die betriebliche Altersversorgung (CEIOPS) eingerichtet. Das Lamfalussy-Verfahren enthielt in dieser ersten Version vier Stufen. ${ }^{37}$ Der Kern war ein ,schlankes ' Gesetzgebungsverfahren, die großzügige Delegation von Befugnissen an die Kommission im Komitologieverfahren, wenn auch zunächst mit ,sunset-clause“ (also zeitlicher Befristung) sowie die Vernetzung der nationalen Aufsichtsbehörden.

Mit der Einführung und Ausweitung des Lamfalussy-Verfahrens geschah in geplanter Weise, was sonst in vielen anderen Kontexten eher ad hoc erfolgt war: Die Komitologie wurde als eine Strategie für eine, wenn man so will, bessere Regulierung eingesetzt, wobei ,besser' hier zum einen ,schneller' heißt, zum anderen, dass die relevanten mitgliedstaatlichen Akteure einbezogen und vernetzt wurden, um Maßnahmen nicht nur auf der europäi-

33 So auch Peter Norman: The Accidental Constitution. The Making of Europe's Constitutional Treaty, Brüssel Euro Comment 2005, S. 84.

34 Europäische Kommission: Mitteilung der Kommission. Finanzdienstleistungen: Umsetzung des Finanzmarktrahmens: Aktionsplan, KOM (1999) 232, S. 16.

35 Ausschuss der Weisen: Schlussbericht des Ausschusses der Weisen über die Regulierung der europäischen Wertpapiermärkte, Brüssel, 15. Februar 2001.

36 Mark A. Pollack: Control Mechanism or Deliberative Democracy? Two Images of Comitology, in: Comparative Political Studies 1/2003, S. 125-155; Europäische Kommission: Europäisches Regieren. Ein Weißbuch, KOM (2001) 428; Alexander Schaub: The Lamfalussy process four years on, in: Journal of Financial Regulation and Compliance 2/2005, S. 110-120.

37 Pollack: Control Mechanism or Deliberative Democracy?, 2003, S. 149-152; Europäische Kommission: Mitteilung der Kommission. Überprüfung des Lamfalussy-Prozesses. Ausbau der aufsichtlichen Konvergenz, KOM (2007) 727; Eva Ruffing: Europäische Wertpapierregulierung zwischen Input- und Output-Legitimität. Das Lamfalussy-Verfahren, Baden-Baden 2011. 
schen Ebene zu verabschieden, sondern auch in den Mitgliedstaaten zügig und möglichst ohne Widerstand umzusetzen. Allerdings war die sehr weitgehende Verlagerung der Regelsetzung auf die Kommission mit Expertenausschüssen schon in der Entstehung umstritten. ${ }^{38}$ Diese Konstellation wurde 2007 und 2011 überarbeitet. In Folge der Finanz- und Wirtschaftskrise wurden die beratenden Ausschüsse (CESR, CEBS und CEIOPS) „zum Fundament einer einheitlichen EU-Finanzaufsichtsarchitektur" ausgebaut ${ }^{39}$ und haben seit Anfang 2011 den Status von Behörden erlangt. ${ }^{40}$

\section{Konvent}

Bereits im Verfassungsentwurf des Konvents von 2003 und dem darauf basierenden Vertrag über die Verfassung der Europäischen Union von 2004, dessen Ratifikation 2005 an den nationalen Referenden in Frankreich und den Niederlanden scheiterte, findet sich die Unterscheidung zwischen delegierter Rechtsetzung einerseits und Durchführungsrechtsakten andererseits. ${ }^{41}$ Ebenfalls findet sich hier schon der Übergang von Ratsbeschlüssen zum ordentlichen Gesetzgebungsverfahren für die Festlegung der neuen Komitologieregeln. ${ }^{42}$ Verfolgt man den Weg zu dieser Regelung, so hat sie ihren Ursprung in der Arbeitsgruppe Vereinfachung des Konvents. Bereits dessen Schlussdokument enthielt die Einführung der delegierten Rechtsetzung, nicht allerdings das Mitentscheidungsverfahren für den Beschluss der Komitologieregeln, ${ }^{43}$ welches erst auf Initiative des Präsidiums eingeführt wurde. ${ }^{44}$ Man weiß bislang nicht genau, wann und wie genau diese Neuerungen, die man durchaus als revolutionär bezeichnen kann, ihren Weg in die Konzepte der Arbeitsgruppe gefunden haben (siehe unten).

\section{Komitologiebeschluss von 2006}

2005 wartete das Parlament angesichts der fehlgeschlagenen Ratifikation des Verfassungsvertrags vergeblich auf eine durchgreifende Reform der Komitologie. ${ }^{45}$ Zudem legte 2006 eine für die Kommission peinliche Dokumentation 50 Fälle offen, in denen die Kommission das Recht des Parlaments, über die laufende Beratung von Durchführungsrechtsakten informiert zu werden, missachtet hatte. ${ }^{46}$ Das Parlament zog erneut die Daumenschrauben an, blockierte die Weiterentwicklung des Lamfalussy-Verfahrens und fror das Budget für die Komitologieausschüsse ein. Überdies begann es in der Gesetzgebungsarbeit systematisch, den Delegationsumfang von Legislativakten zu beschränken. ${ }^{47}$ Aufgrund dieses vom Europäischen Parlament aufgebauten Drucks wurde schließlich durch Ratsbeschluss das

38 Pollack: Control Mechanism or Deliberative Democracy?, 2003, S. 150-151.

39 Norbert Walter/Steffen Kern: Politikberatung im EU Finanzbinnenmarkt - Vom Lamfalussy-Prozess zur europäischen Aufsicht, in: Vierteljahreshefte zur Wirtschaftsforschung 1/2011, S. 91-105.

40 Hustedt et al.: Verwaltungsstrukturen in der Europäischen Union, 2013, hier Kap. 5.

41 Artt. 35 und 36 des Entwurfs für einen Vertrag über eine Verfassung für Europa, CONV 850/03 beziehungsweise Artt. I-36 und I-37 des Vertrags über die Verfassung für Europa.

42 Andreas Maurer: Orientierungen im Vefahrensdickicht? Die neue Normenhierarchie der Europäischen Integration, in: integration 4/2003, S. 440-453, hier S. 443-444.

43 Europäischer Konvent: Bericht des Vorsitzenden der Gruppe IX „,Vereinfachung“ an die Mitglieder des Konvents, CONV 424/02, S. 10-12.

44 Héritier et al: Changing Roles of Delegation, 2013, S. 40.

45 Christiansen/Dobbels: Comitology and delegated acts after Lisbon, 2012, S. 7.

46 Kieran St. Clair Bradley: Halfway house: The 2006 comitology reforms and the European Parliament, in: West European Politics 4/2008, S. 837-854.

47 Héritier et al: Changing Roles of Delegation, 2013, S. 42-44. 
Regelungsverfahren mit Kontrolle eingeführt. ${ }^{48}$ Dieses Verfahren sollte eingesetzt werden, wenn im Mitentscheidungsverfahren (also unter gleichberechtigter Beteiligung von Rat und Europäischem Parlament) Durchführungsbefugnisse an die Kommission delegiert werden, die die Verabschiedung von Maßnahmen von allgemeiner Tragweite vorsehen. In diesem Verfahren konnte der Gesetzgeber (also Rat und Parlament) nun die Verabschiedung einer Maßnahme ablehnen und zwar mit der Begründung, dass die Maßnahme über die delegierten Befugnisse hinausgeht, mit dem Ziel und Inhalt des Basisrechtsaktes nicht vereinbar ist oder gegen die Grundsätze der Subsidiarität oder Verhältnismäßigkeit verstößt. ${ }^{49}$ Ein Rückruf aus anderen, politisch motivierten Gründen blieb aber weiter verwehrt. Eine Vielzahl von Rechtsakten wurde in der Folgezeit hinsichtlich der Komitologieverfahren entsprechend angepasst. ${ }^{50}$ In der Praxis war die Neigung von Rat und Parlament, diese Möglichkeit wahrzunehmen, unterschiedlich ausgeprägt: 2008 legten Rat oder Parlament bei sieben Maßnahmen ein Veto ein, 2009 war es nur eine Maßnahme, die das Europäische Parlament ablehnte. ${ }^{51}$ Aber wie alle formalen Verfahren dürfte die Regelung bereits im Vorfeld gewirkt und die Kommission motiviert haben, schon vorab (informell) auch die Positionen des Europäischen Parlaments zu berücksichtigen.

\section{Komitologie nach ,Lissabon' - oder was von ihr übrig ist}

Im Folgenden wird zunächst die Unterscheidung zwischen den beiden Rechtsetzungsverfahren im Primärrecht nach Artt. 290 und 291 AEUV betrachtet. Dann werden die Maßnahmen zu deren Konkretisierung (Common Understanding und Komitologieverordnung) vorgestellt, die im Laufe des Jahres 2010 als Paket verhandelt wurden.

\section{Artt. 290 und 291 AEUV}

Die bereits im Konventsentwurf enthaltene Unterscheidung zwischen delegierter Rechtsetzung und Implementationsmaßnahmen, wobei nur bei Letzterer die Komitologie zum Einsatz kommt, findet sich im AEUV in den Artt. 290 und 291 im Wesentlichen genauso wieder. Unterschieden werden hier genau genommen drei Normenkategorien unterhalb des Primärrechts: ${ }^{52}$

- ordentliche, im Gesetzgebungsverfahren zustande gekommene Gesetze (Art. 289 AEUV);

- „,delegierte Rechtsakte“ (Art. 290 AEUV); und

- „Durchführungsrechtsakte“ (Art. 291 AEUV).

Die Komitologie - und das ist eine gravierende Neuerung - kommt nur noch bei den Durchführungsmaßnahmen nach Art. 291 AEUV zum Einsatz, während die delegierten

48 Beschluss des Rates vom 17. Juli 2006 zur Änderung des Beschlusses 1999/468/EG zur Festlegung der Modalitäten für die Ausübung der der Kommission übertragenen Durchführungsbefugnisse, in: Amtsblatt der EU, Nr. L 200 vom 5. Mai 2006, S. 11.

49 Christine Neuhold: Taming the 'Trojan Horse' of Comitology? Accountability issues of Comitology and the Role of the European Parliament, European Integration online Papers 1/2008; Dieter Wolfram: „Underground Law"? Abgeleitete Rechtsetzung durch Komitologieverfahren in der EU: Bedeutung, Stand und Aussichten nach dem Vertrag von Lissabon, Centrum für Europäische Politik: CEP Studie 2009.

50 Europäische Kommission: Mitteilung der Kommission an das Europäische Parlament und den Rat. Umsetzung von Artikel 290 des Vertrags über die Arbeitsweise der Europäischen Union, KOM (2009) 673, S. 2.

51 Europäische Kommission: Bericht der Kommission über die Tätigkeit der Ausschüsse im Jahre 2009, KOM (2010) 354 .

52 Herwig C. Hofmann: Legislation, Delegation and Implementation under the Treaty of Lisbon: Typology Meets Reality, in: European Law Journal 4/2009, S. 482-505, hier S. 487-494. 
Rechtsakte von der Kommission alleine verabschiedet, aber von Parlament und Rat als delegierendem Gesetzgeber ex post kontrolliert werden.

Mit Art. 290 AEUV wird erstmals eine ganz neue Kategorie der Rechtsetzung eingeführt, für die die Kommission alleine (ohne Komitologieausschuss) zuständig ist. Mit diesen Rechtsakten dürfen nicht-wesentliche Vorschriften des Basisrechtsakts ergänzt oder geändert werden. Im Basisrechtsakt müssen zudem Ziele, Inhalt, Geltungsbereich und Dauer der Befugnisübertragung ausdrücklich festgelegt werden. Sowohl bei der Referenz zur Wesentlichkeit als auch bei der Pflicht zur Präzisierung der Übertragung schimmern deutsche Verfassungskonzepte ${ }^{53}$ auf. Für diese neue Kategorie von Rechtsakten kann im Legislativakt eines von zwei Kontrollverfahren festgelegt werden: entweder können Europäisches Parlament oder Rat jeweils beschließen, die Übertragung zu widerrufen, oder der delegierte Rechtsakt kann nur in Kraft treten, wenn das Europäische Parlament oder der Rat innerhalb einer Frist keine Einwände erhebt. Parlament und Rat haben hier also endlich gleiche Rechte. Weil Art. 290 AEUV ein vollständig neues Verfahren einführte, vereinbarten die Institutionen ein Common Understanding (siehe unten), auch wenn rechtlich gesehen Art. 290 AEUV im Gegensatz zu Art. 291 AEUV keiner weiteren Regelung bedarf.

Art. 291 AEUV stellt zunächst fest, dass die Durchführung des Gemeinschaftsrechts Aufgabe der Mitgliedstaaten ist und dass der Kommission, in begründeten Sonderfällen auch dem Rat, Durchführungsbefugnisse übertragen werden können, wenn dies erforderlich ist. Für die Regeln und Grundsätze der Komitologie wird sodann das ordentliche Gesetzgebungsverfahren festgelegt.

Die zentrale Frage, wie man genau Rechtsakte nach Art. 290 AEUV und solche nach Art. 291 AEUV unterscheidet, ist sowohl in der rechtswissenschaftlichen Literatur als auch in der Praxis sehr umstritten. Die Formulierungen der Artt. 290 und 291 AEUV geben nach Auffassung verschiedener rechtswissenschaftlicher Analysen keine klaren Kriterien vor. Schon die Formulierung beider Normen sei ganz unterschiedlich, ${ }^{54}$,da der Begriff delegierter Rechtsakt auf die Verankerung im Basisrechtsakt verweist, während der Begriff Durchführungsrechtsakt sich an der Funktion des Rechtsaktes orientiert". ${ }^{55}$ Dreh- und Angelpunkt ist letztlich die Frage, wann Elemente des Basisrechtsaktes „ergänzt oder geändert“ werden. ${ }^{56}$ Die Zuordnung von Maßnahmen von legislativem, abstrakt-generellem Charakter zu Art. 290 AEUV $^{57}$ und Maßnahmen von exekutivem, ggf. konkret-individuellem Charakter zu Art. 291 AEUV nutzt jedenfalls im Grenzfall wenig. ${ }^{58}$ Dies wird auch deutlich in der Stellungnahme des Auswärtigen Amtes zu diesen Neuerungen. Im Abschnitt zu den Durchführungsrechtsakten heißt es: „Es verbleibt allerdings ein gewisser Überschneidungsbereich hinsichtlich der Ergänzung des zugrundeliegenden Gesetzgebungsakts. Hier hat der europäische Gesetzgeber einen Beurteilungsspielraum, welches Instrument er für angemessen

53 Art. 80 Abs. 1 S. 2 Grundgesetz.

54 Hofmann: Legislation, Delegation and Implementation, 2009, S. 496.

55 Daniel Scharf: Das Komitologieverfahren nach dem Vertrag von Lissabon - Neuerungen und Auswirkungen auf die Gemeinsame Handelspolitik, in: Universität Halle-Wittenberg: Beiträge zum Transnationalen Wirtschaftsrecht, Heft 101, 2010; auch Wolfram: „Underground Law?“, 2009; Hofmann et al: Administrative Law and Policy of the European Law Journal, 2011, S. 533.

56 Paul Craig: Delegated Acts, Implementing Acts and the New Comitology Regulation, in: European Law Review 5/2011, S. 671-687, hier S. 672-674.

57 Delegierte Rechtsetzung adressiert demnach legislative Fragen in einem nicht-legislativen Verfahren. Vgl. Hofmann: Legislation, Delegation and Implementation, 2009.

58 Scharf: Das Komitologieverfahren nach dem Vertrag von Lissabon, 2010; schon Herwig C. Hofmann/Annette E. Töller: Zur Reform der Komitologie - Regeln und Grundsätze für die Verwaltungskooperation im Ausschusssystem der Europäischen Gemeinschaft, in: Staatswissenschaften und Staatspraxis 2/1998, S. 209-239. 
hält. “59 Schließlich können die verschiedenen Normkategorien vor dem Hintergrund verschiedener Konzepte des Mehrebenensystems ganz unterschiedlich interpretiert werden. ${ }^{60}$

Dabei ist die delegierte Rechtsetzung nach Art. 290 AEUV eben nicht nur als Versuch zu sehen, Dinge von größerer Tragweite nicht mehr im Kontext der Komitologie wahrzunehmen, sondern auch (analog zum oben angesprochenen Lamfalussy-Verfahren) als Anreiz für den Gesetzgeber, weniger detailliert zu regeln und mehr Regelungsbefugnisse an die Kommission zu delegieren, wobei (unter interessantem Rückgriff auf die Terminologie des Bundesverfassungsgerichts) allerdings die Regelung wesentlicher Aspekte eines Bereichs im Gesetzgebungsakt nach Art. 289 AEUV erfolgen muss und nicht gem. Art. 290 AEUV delegiert werden darf.

Die Unterscheidung zwischen Artt. 290 und 291 AEUV ist nicht nur rechtsdogmatisch von Interesse, sondern angesichts der unterschiedlichen Verfahrensweisen (einmal mit Kontrolle durch den Gesetzgeber und einmal durch die Komitologie) und der damit einhergehenden verschiedenen Ressourcen für verschiedene Institutionen politisch höchst brisant. ${ }^{61}$ Daher ist in dieser Frage mit weiteren Konflikten zwischen Kommission, Parlament und Rat im Gesetzgebungsverfahren zu rechnen.

\section{Tabelle 1: Die neuen Verfahren unterhalb der Gesetzgebung im AEUV}

\begin{tabular}{|l|l|l|}
\hline & \multicolumn{1}{|c|}{,Delegierte Rechtsakte } & \multicolumn{1}{|c|}{,Durchführungsrechtsakte } \\
\hline Vertragsgrundlage & Art. 290 AEUV & Art. 291 AEUV \\
\hline Konkretisierung & Common Understanding 2011 & Komitologieverordnung 2011 \\
\hline Verfahren & $\begin{array}{l}\text { Alleiniges Entscheidungsrecht } \\
\text { der Kommission, Revokations- } \\
\text { rechte von Europäischem Parla- } \\
\text { ment und Rat in zwei Varianten }\end{array}$ & $\begin{array}{l}\text { Kommissionsentscheidung } \\
\text { nach Befassung der Komitolo- } \\
\text { gie } \\
\text { - Beratungsverfahren } \\
\text { - Prüfverfahren } \\
\text { - gegebenenfalls weitere Ver- } \\
\text { fahrensoptionen }\end{array}$ \\
\hline Gegenstände & $\begin{array}{l}\text { Legislative Ergänzung nicht-we- } \\
\text { sentlicher Inhalte des Basis- } \\
\text { rechtsakts; } \\
\text { abstrakt-generelle Maßnahmen }\end{array}$ & $\begin{array}{l}\text { Exekutive Durchführungsmaß- } \\
\text { nahme; } \\
\text { Einzelfallentscheidungen }\end{array}$ \\
\hline
\end{tabular}

Quelle: eigene Darstellung.

59 Auswärtiges Amt: Denkschrift zum Vertrag von Lissabon, 2007, AS-RK 2007. Ob allerdings die Unterscheidung so klar ist, dass eine ,falsche“ Zuweisung von Materien in Artt. 290 oder 291 AEUV durch den Gesetzgeber dazu führt, dass ein Rechtsakt und auf seiner Basis zustande gekommene delegierte oder Durchführungsrechtsakte illegal sind, darf man bezweifeln. Vgl. Buchet et al.: Changing Rules of Delegation, 2012.

60 Man kann, wie Hofmann aufzeigt, die neue Konstellation insgesamt als Kompromiss zwischen den Institutionen (also auf einer horizontalen Ebene) betrachten. Man kann sie aber auch im Sinne eines Exekutivföderalismus so verstehen, dass Art. 290 AEUV die Delegation legislativer Materien durch den föderalen Gesetzgeber betrifft, Art. 291 AEUV jedoch - unter der Prämisse, dass die Durchführung den Mitgliedstaaten obliegt - das vertikale Verhältnis Gemeinschaft-Mitgliedstaaten betrifft. Vgl. Hofmann: Legislation, Delegation and Implementation, 2009, S. 497-498.

61 Vgl. zum Beispiel Scharf: Das Komitologieverfahren nach dem Vertrag von Lissabon, 2010, S. 21; Rudolf Geiger/Daniel-Erasmus Khan/Markus Kotzur: EUV/AEUV. Vertrag über die Europäische Union und Vertrag über die Arbeitsweise der Europäischen Union, München 2010, S. 868; Vihar Georgiev: Commission on the Loose? Delegated Lawmaking and Comitology after Lisbon. Paper prepared for the EUSA Twelfth Biennial International Conference, Boston, Massachusetts, 3.-5. März, Hyatt Regency Boston 2011, S. 15; Craig: Delegated Acts, 2011, S. 677. 


\section{Common Understanding}

Im Gegensatz zu Art. 291 AEUV, der einer neuen Regelung zur Komitologie bedurfte, wäre Art. 290 AEUV im Prinzip ohne Konkretisierung anwendbar gewesen. Die Institutionen verständigten sich dennoch darauf, ein Common Understanding zu entwickeln, das als Paket mit der Komitologieverordnung verhandelt wurde. Bei der Verhandlung des Common Understanding ging es zunächst um die Dauer der Delegation. ${ }^{62}$ Eine zentrale Kontroverse war zudem die Einbeziehung von Experten bei der Entwicklung delegierter Rechtsakte. Während die Kommission sich nicht gerne zu solchen Konsultationen verpflichten lassen wollte (sie sah diese als Einführung der Komitologie durch die Hintertür) und Wert darauf legte, dass es hier um Konsultation und nicht um Verhandlungen oder Veränderung ihrer Vorschläge geht, ${ }^{63}$ wollten die Mitgliedstaaten, dass in jedem Rechtsakt, der delegierte Rechtsetzung vorsieht, festgelegt wird, dass die Kommission nationale Experten aus allen Mitgliedstaaten konsultieren muss. Das Parlament wollte eine Expertenkonsultation nur akzeptieren, wenn nicht nur nationale, sondern auch seine Experten gehört werden sollten. Auch der Zeitpunkt der Konsultation erwies sich als strittig. ${ }^{64}$ Hinter der Diskussion über die Expertenkonsultation steckt ein gewisses Widerstreben der Mitgliedstaaten, sich ganz von den Komitologiestrukturen und ihrer gut eingespielten Scharnierfunktion zwischen Kommission und mitgliedstaatlichen Verwaltungen ${ }^{65}$ zu trennen. Zudem bedeutet die Konsultation von Experten für Mitgliedstaaten und Parlament auch die Möglichkeit, über die Entwicklung solcher Maßnahmen rechtzeitig informiert zu sein, was eine Voraussetzung für die sinnvolle Nutzung der Kontrollverfahren ist. Der schließlich erst im Frühjahr 2011 verabschiedete Text des Common Understanding enthält die Formulierung: „The Commission, when preparing and drawing up delegated acts, will [...] carry out appropriate and transparent consultations well in advance, including at expert level." "66 Des Weiteren sind hier Fristen und Bedingungen für die Übermittlung von Dokumenten durch die Kommission sowie für die Einreichung von Widersprüchen durch Rat und Parlament festgelegt. Solche Fristen sind vor allem für das Europäische Parlament wichtig, weil vor einer Beschlussfassung die Befassung eines Ausschusses und des Plenums erforderlich ist. ${ }^{67}$

\section{Komitologieverordnung von 2011}

Die Kommission konnte sich mit vielen ihrer Vorstellungen zur Komitologie, die ihr weitgehende Spielräume gesichert hätten, nicht durchsetzen. Ihr 2010 unterbreiteter Vorschlag erwies sich als kaum mehr als eine Diskussionsgrundlage. ${ }^{68}$ Besonders umstritten war die Ausgestaltung des Berufungsausschusses, dessen Befassung an die Stelle des bisherigen Rekurses zum Rat treten sollte. Dem Parlament war vor allem der vorgesehene Vorsitz durch einen Vertreter der Mitgliedstaaten ein Dorn im Auge. Des Weiteren umstritten waren

62 Siehe im Einzelnen Brandsma/Blom-Hansen: Negotiating the Post-Lisbon Comitology System, 2012, S. 946.

63 Buchet et al.: Changing Rules of Delegation, 2012, Min. 41:30.

64 Christiansen/Dobbels: Comitology and delegated acts after Lisbon, 2012, S. 9; Brandsma/Blom-Hansen: Negotiating the Post-Lisbon Comitology System, 2012, S. 946-947.

65 Hustedt et al.: Verwaltungsstrukturen in der Europäischen Union, 2013, Kap. 4.

66 Rat der Europäischen Union: Common Understanding - Delegated Acts, Dok. 8753/11, S. 4.

67 Brandsma/Blom-Hansen: Negotiating the Post-Lisbon Comitology System, 2012, S. 945.

68 Europäische Kommission: Vorschlag für eine Verordnung des Europäischen Parlaments und des Rates zur Festlegung der allgemeinen Regeln und Grundsätze, nach denen die Mitgliedstaaten die Wahrnehmung der Durchführungsbefugnisse durch die Kommission kontrollieren, KOM (2010) 83; Christiansen/Dobbels: Comitology and delegated acts after Lisbon, 2012, S. 10; im Einzelnen siehe Brandsma/Blom-Hansen: Negotiating the Post-Lisbon Comitology System, 2012, S. 948-952. 
Schutzklauselverfahren im Bereich der Handelspolitik und die Abstimmungsmodalitäten in besonders sensiblen Angelegenheiten. Nach Einschätzung von Christiansen und Dobbels konnte das Europäische Parlament nur hinsichtlich des Vorsitzes einen Erfolg vermelden: dieser liegt nun bei der Kommission. ${ }^{69}$ Hinsichtlich einer ganzen Reihe anderer Punkte (zum Beispiel der automatischen Anpassung des bestehenden Acquis an die neuen Verfahren und des Beobachterstatus' des Parlaments im Berufungsausschuss) konnte sich das Parlament jedoch nicht durchsetzen. ${ }^{70}$ Allerdings unterstützte es ausdrücklich die Idee der Kommission, den Rekurs durch den Berufungsausschuss zu ersetzen. Alles in allem überwiegen bei der konkreten Ausgestaltung der Komitologie Elemente der Kontinuität gegenüber dem alten Komitologiesystem. ${ }^{71}$ Inwiefern tatsächlich eine Vereinfachung der Verfahren erreicht wurde, ist allerding umstritten. ${ }^{72}$

Ein wesentliches Element der neuen Komitologieverordnung ${ }^{73}$ ist die Reduzierung der Verfahren auf - im Prinzip - nur noch zwei: Im Beratungsverfahren muss die Kommission (wie beim bisherigen beratenden Ausschuss) den Ausschuss konsultieren, soll dessen Stellungnahme so weit wie möglich berücksichtigen, muss ihr aber nicht folgen. ${ }^{74}$ Im Prüfverfahren kann die Kommission (ähnlich wie beim bisherigen Verwaltungsverfahren) nur dann eine Maßnahme nicht erlassen, wenn im Ausschuss eine qualifizierte Mehrheit dagegen zustande kommt. ${ }^{75}$ In diesem Fall kann die Kommission die Maßnahme zurückziehen, dem Ausschuss einen geänderten Vorschlag oder die Maßnahme dem Berufungsausschuss vorlegen. ${ }^{76}$ Die Befassung des aus Vertretern der Mitgliedstaaten zusammengesetzten Berufungsausschusses ersetzt den Rekurs zum Rat, der abgeschafft wird. ${ }^{77}$ Analog zum Komitologiebeschluss von 1999 können bei der Durchführung von im ordentlichen Gesetzgebungsverfahren beschlossenen Basisrechtsakten Rat und Parlament hier (nur) überwachen, ob die Durchführungsmaßnahme die delegierten Befugnisse überschreitet. ${ }^{78}$ Allerdings öffnet Art. 5 Abs. 4 S. 2 Komitologieverordnung ein ,Hintertürchen“ für ein weiteres Verfahren (analog zum bisherigen Regelungsverfahren): In diesem kann die Kommission in bestimmten, für sensibel erachteten Gebieten (Besteuerung, Finanzdienstleistungen, Schutz der Gesundheit oder Sicherheit von Menschen, Tieren und Pflanzen oder endgültige multilaterale Schutzmaßnahmen) nur dann eine Maßnahme erlassen, wenn sie eine qualifizierte Mehrheit im Ausschuss für die Maßnahmen erhält oder keine einfache Mehrheit dagegen. Art. 5 Abs. 5 Komitologieverordnung sieht eine weitere Ausnahme für Antidumping- oder Aus-

69 Christiansen/Dobbels: Comitology and delegated acts after Lisbon, 2012, S. 11; im Einzelnen siehe Brandsma/ Blom-Hansen: Negotiating the Post-Lisbon Comitology System, 2012, S. 951.

70 Christiansen/Dobbels: Comitology and delegated acts after Lisbon, 2012, S. 13-15.

71 Brandsma/Blom-Hansen: Negotiating the Post-Lisbon Comitology System, 2012, S. 954-955.

72 Craig: Delegated Acts, 2011, S. 684-685.

73 Verordnung (EU) Nr. 182/2011 des Europäischen Parlaments und des Rates vom 16. Februar 2011 zur Festlegung der allgemeinen Regeln und Grundsätze, nach denen die Mitgliedstaaten die Wahrnehmung der Durchführungsbefugnisse durch die Kommission kontrollieren, in: Amtsblatt der EU, Nr. L 55 vom 28. Februar 2011, S. 13-18.

74 Art. 4 Komitologieverordnung; Craig: Delegated Acts, 2011, hier S. 679.

75 Art. 5 Abs. 3 Komitologieverordnung. Es gelten analog die Regeln für den Rat, wonach eine qualifizierte Mehrheit ab dem 1. November 2014 gem. Art. 16 Abs. 4 EUV erreicht ist, wenn ,,mindestens 55\% der Mitglieder des Rates, gebildet aus 15 Mitgliedern, sofern diese von den vertretenden Mitgliedstaaten mindestens $65 \%$ der Bevölkerung der Union ausmachen“" zustimmen. Bis 2014 gilt Art. 3 Abs. 3 Protokoll Nr. 36 über die Übergangsbestimmungen.

76 Art. 6 Komitologieverordnung.

77 Christiansen/Dobbels: Non-Legislative Rule Making after the Lisbon Treaty, 2013, hier S. 48-49.

78 Art. 5 Komitologieverordnung; Craig: Delegated Acts, 2011, S. 678. 
gleichsmaßnahmen vor, Art. 7 Komitologieverordnung regelt Ausnahmefälle, in denen schnell entschieden werden muss. ${ }^{79}$

Des Weiteren legt die Komitologieverordnung Kriterien fest, welche Verfahren in welchen Politikfeldern und für welche Aufgaben verwendet werden sollen: ${ }^{80}$ So soll das Prüfverfahren bei Maßnahmen von ,allgemeiner Tragweite“ sowie bei allen Durchführungsrechtsakten in Bezug auf Programme mit wesentlichen Auswirkungen, die gemeinsame Agrar- und Fischereipolitik, den Umwelt- und Gesundheitsschutz, die Handelspolitik sowie die Besteuerung verwendet werden. Neu ist auch, dass die Komitologie nun auch in der Handelspolitik anwendbar ist.

Die Komitologieverordnung sieht in Art. 13 eine automatische Anpassung der Ausschussverfahren bei bereits bestehenden Rechtakten vor. Dabei wird das frühere Beratungsverfahren zum neuen Beratungsverfahren, die früheren Verwaltungs- und Regelungsverfahren werden zu Prüfverfahren und das frühere Verfahren bei Schutzmaßnahmen wird zum Dringlichkeitsverfahren. ${ }^{81}$ Lediglich bei Regelungsverfahren mit Kontrolle wird nicht automatisch angepasst, sondern die Rechtsakte müssen geändert werden. ${ }^{82}$ Es muss von Fall zu Fall entschieden werden, ob die Materien unter Artt. 290 oder 291 AEUV fallen. 2016 muss die Kommission einen Bericht über die Durchführung der Komitologieverordnung vorlegen. ${ }^{83}$

\section{Voraussichtliche Effekte der Reform}

Auch wenn im Verfahren nach Art. 290 AEUV heute nicht nur Materien geregelt werden, die zuvor nach Art. 202 EGV im Komitologieverfahren entschieden wurden, sondern (jedenfalls der Intention nach) auch solche, die zuvor im Gesetzgebungsverfahren beschlossen wurden, ${ }^{84}$ dürfte die Einführung der delegierten Gesetzgebung im Ergebnis zu einer deutlichen Beschneidung des Wirkungsgebietes der Komitologie führen. Zwar wird sich dies nicht in einer Abnahme der Anzahl der Ausschüsse zeigen, aber diese werden in der Substanz weniger zu entscheiden haben und auch weniger Entscheidungen treffen.

In der Literatur wird gegenwärtig rege diskutiert, wer der Gewinner der Reform ist. ${ }^{85} \mathrm{Da}$ die Vorschläge des Konvents, wie sie sich seit 2009 im Primärrecht wiederfinden, in vielen Punkten die lange vertretenen Positionen des Europäischen Parlaments weitgehend umgesetzt haben, galt zunächst das Parlament als Gewinner. Angesichts des eher bescheidenen Erfolgs des Parlaments bei der Komitologieverordnung wird dies inzwischen von vielen Autoren bezweifelt. ${ }^{86}$ Es überwiegen nun die Beobachter, die die Kommission als Gewinnerin der neuen Rechtslage sehen. In der neuen Komitologie habe sie nun mehr Macht, da kein Rekurs zum Rat mehr drohe. ${ }^{87}$ Bei den Kompetenzen nach Art. 290 AEUV könne die Kommission nun ganz ohne Kontrolle durch die Komitologieausschüsse agieren, während

79 Zu den Einzelheiten: Ebenda, S. 680-681.

80 Art. 2 Komitologieverordnung.

81 Europäische Kommission: Bericht der Kommission über die Tätigkeit der Ausschüsse im Jahr 2011, KOM (2012) 685, S. 4; kritisch zu dieser automatischen Überleitung: Craig: Delegated Acts, 2011, S. 676.

82 Christiansen/Dobbels: Non Legislative Rule Making after the Lisbon Treaty, 2013, S. 45.

83 Art. 15 Komitologieverordnung.

84 Siehe Liisberg: The EU Constitutional Treaty and its distinction, 2006, S. 44.

85 Vgl. zum Beispiel Edenharter: Die Komitologie nach dem Vertrag von Lissabon, 2011; Georgiev: Commission on the Loose?, 2011; Christiansen/Dobbels: Comitology and delegated acts after Lisbon, 2012, S. 3; Ritleng: La délégation du pouvoir legislatif de 1'Union Européenne, 2010, S. 573-574.

86 Christiansen/Dobbels: Comitology and delegated acts after Lisbon, 2012.

87 Edenharter: Die Komitlogie nach dem Vertrag von Lissabon, 2011; Greorgiev: Commission on the Loose, 2011; Christiansen/Dobbels: Non-Legislative Rule Making after the Lisbon Treaty, 2013, S. 44; Buchet et al.: Changing Rules of Delegation, 2012, Min. 50:00. 
die Nutzung des damit verbundenen Revokationsrechts durch das Europäische Parlament und den Rat erst im Anschluss an den eigentlichen Entscheidungsprozess stattfinde. Auch kann auf der Basis von Art. 290 AEUV mit einer großzügigeren Delegation von Kompetenzen durch den Gesetzgeber gerechnet werden. Allerdings zwingt die neue Konstellation die Kommission, in ihren Gesetzentwürfen sehr genau zu definieren und gegenüber dem Gesetzgeber zu begründen, für was und warum sie Kompetenzen delegiert haben möchte. ${ }^{88}$

Zugleich ist aber noch offen, wie sich die Institutionen längerfristig zu den neuen Verfahren verhalten werden. Die relativ unklare Unterscheidung zwischen Artt. 290 und 291 AEUV eröffnet grundsätzlich neue Räume für Konflikte, insbesondere zwischen Parlament und Rat. Es kann plausibel angenommen werden, dass in der Gesetzgebung das Europäische Parlament prinzipiell ein Interesse daran hat, möglichst viele Materien gem. Art. 290 AEUV zu delegieren, weil es hier gleichberechtigt neben dem Rat kontrolliert, während die Kommission in der Entscheidungsphase ohne privilegierten Einfluss der Mitgliedstaaten über die Komitologieausschüsse agiert. ${ }^{89}$ Hingegen dürfte der Rat daran interessiert sein, Materien gem. Art. 291 AEUV zu delegieren, weil hier nach wie vor mitgliedstaatliche Delegierte in den Ausschüssen partizipieren.

Allerdings ist gegenwärtig noch unklar, welche Relevanz das Parlament diesem Thema beimisst und wie viel Wert es im Einzelfall auf diese institutionellen Finessen legt, wenn es ja immer auch Konflikte über substanzielle Inhalte der Rechtsetzung gibt. ${ }^{90}$ Zumindest der Rat scheint entschlossen, so wenig wie möglich gem. Art. 290 AEUV zu delegieren. Selbst wenn das Parlament im Rahmen von Paketlösungen hier mitspielen sollte, wird sich die Kommission wohl früher oder später mit rechtlichen Mitteln wehren. ${ }^{91}$

Schließlich ist auch die Überführung des Acquis noch nicht abgeschlossen und wird bis zu ihrem Abschluss 2014 voraussichtlich Quelle für neue Konflikte sein. ${ }^{92}$ Während sich Parlament und Rat einig sind, dass die Bereiche, in denen bisher das Regelungsverfahren mit Kontrolle Anwendung fand, nicht (wie von der Kommission vorgeschlagen) automatisch in delegierte Rechtsetzung überführt werden sollen, sind sich beide auch hier zutiefst uneinig über die Art der Abweichung: Während das Parlament das Anwendungsgebiet der delegierten Rechtsetzung gerne breiter sehen würde, vertritt der Rat die gegenteilige Position. ${ }^{93}$

\section{Erklärung des radikalen Wandels}

Die vorangegangenen Ausführungen dürften verdeutlicht haben, wie gravierend die Reformen des Lissaboner Vertrags im Bereich der Delegation von Regelungsbefugnissen auf die Kommission sind. Insbesondere vor dem Hintergrund der vorangegangenen 20 Jahre, in denen es trotz massiver institutioneller Konflikte allenfalls zu inkrementellen Reformen gekommen war, ${ }^{94}$ stellt sich die Frage, wie es möglich war, dass in diesem Bereich eine derart gravierende Veränderung beschlossen wurde. Die Rezeption der Entwicklung in der Literatur gibt bislang nur Bruchteile einer Antwort auf diese Frage. Dabei sind vor allem institutio-

88 Ebenda, Min. 34:30.

89 Brandsma/Blom-Hansen: Negotiating the Post-Lisbon Comitology System, 2012, S. 942.

90 Christiansen/Dobbels: Comitology and delegated acts after Lisbon, 2012, S. 16.

91 Buchet et al: Changing Rules of Delegation, 2012, Min. 45:00.

92 Christiansen/Dobbels: Comitology and delegated acts after Lisbon, 2012, S. 17; Brandsma/Blom-Hansen: Negotiating the Post-Lisbon Comitology System, 2012, S. 955.

93 Christiansen/Dobbels: Comitology and delegated acts after Lisbon, 2012, S. 17.

94 Aufgrund der Machtposition im Rat, vgl. zum Beispiel Brandsma/Blom-Hansen: Negotiating the Post-Lisbon Comitology System, 2012, S. 940. 
nentheoretische Ansätze zur Anwendung gekommen. Diese betonen prinzipiell, dass Institutionen das Handeln von Akteuren beeinflussen, indem sie etwa Handlungsressourcen zuweisen, aber gegebenenfalls auch beeinflussen, wie Akteure ihre Präferenzen wahrnehmen. Die Politikwissenschaft kennt drei verschiedene institutionentheoretische Ansätze, die aus recht verschiedenen epistemologischen Kontexten stammen und auch mit sehr unterschiedlichen Grundannahmen arbeiten. Der Historische Institutionalismus betont insbesondere institutionelle Pfadabhängigkeiten und nichtintendierte Konsequenzen. ${ }^{95}$ Der RationalChoice-Institutionalismus geht davon aus, dass Institutionen das Kalkül von rationalen, strategisch handelnden Akteuren beeinflussen und ihnen Ressourcen zuweisen..$^{96}$ Er wurde bislang besonders gerne zur Erklärung der Entstehung und der Effekte unterschiedlicher institutioneller Regeln in der Europäischen Union herangezogen. Er geht hier davon aus, dass verschiedene Akteure klar angebbare Interessen an bestimmten institutionellen Regelungen haben und - je nach Ressourcenausstattung durch die bereits bestehenden Verfahren - unterschiedlich gut in der Lage sind, diese Interessen durchzusetzen. ${ }^{97}$ Der Soziologische Institutionalimus hingegen betont die Rolle kultureller Faktoren bei der Entstehung, Entwicklung und Nutzung von Institutionen. ${ }^{98}$

Im Folgenden soll gezeigt werden, dass man einige der aufgezeigten Entwicklungen anhand der bislang vorliegenden Daten noch gar nicht erklären kann, dass man aber schon deutlich weiterkommt, wenn man neben rational-choice-institutionalistischen auch historisch-institutionalistische und soziologisch-institutionalistische Erklärungsansätze mit einbezieht.

Betrachten wir zunächst die Ebene der Vertragsrevision, so sind weder Europäisches Parlament noch Kommission zentrale Spieler, auch wenn beide im Vorfeld von Regierungskonferenzen regelmäßig mit Berichten und Positionen in Erscheinung getreten sind. Das Standard-Verfahren der Vertragsänderungen war (und ist) das der Regierungskonferenzen, auf denen an jeweiligen nationalen Interessen orientierte Regierungsvertreter im Modus der Einstimmigkeit Einigungen auf dem kleinsten gemeinsamen Nenner hervorbrachten und -bringen. ${ }^{99}$ Die Konventsmethode ist unter anderem eingeführt worden, um durch die Einbeziehung weiterer Akteure (Vertreter nationaler Parlamente, des Europäischen Parlaments und der Kommission) und die Bildung von Arbeitsgruppen ein anderes Verfahren der Willensbildung zu etablieren. Im Gegensatz zum ,bargaining' der Regierungskonferenzen sollte die Konventsmethode einen gleichermaßen sachorientierten wie auch an die Öffentlichkeit adressierten, deliberativen Dialog über die geplante europäische Verfassung ermöglichen. ${ }^{100}$ Bei allem Neuen stellte der Konvent aber keine fundamentale Veränderung der bisherigen Methode der Vertragsänderung dar, sondern war lediglich eine Ergänzung. ${ }^{101} \mathrm{Im}$ Konvent erhielten Parlament und Kommission Zugang zu den Beratungen, auch in der von Konvents-Vizepräsident und Verfassungsrechtler Giuliano Amato geleiteten Arbeitsgruppe Vereinfachung, die sich unter anderem mit der Frage befassen musste, ob eine Unterschei-

95 Peter A. Hall/Rosemary C. R. Taylor: Political Science and the Three New Institutionalisms, Max-PlanckInstitut für Gesellschaftsforschung: MPIFG Discussion Paper 96/6, S. 7.

96 Ebenda, 1996, S. 12.

97 Ebenda, S. 10-13; für die Komitologie: Brandsma/Blom-Hansen: Negotiating the Post-Lisbon Comitology System, 2012, S. 940; Héritier et al.: Changing Rules of Delegation, 2013, S. 14-16.

98 Hall/Taylor: Political Science and the Three New Institutionalisms, 1996, S. 14-17.

99 Maurer: Deliberation und Bargaining im Konvent, 2006, S. 3.

100 Norman: The Accidental Constitution, 2005, S. 107; Maurer: Deliberation und Bargaining im Konvent, 2006, S. 3 .

101 Ebenda, S. 4. 
dung zwischen exekutiven und legislativen Maßnahmen eingeführt werden solle. In der Erklärung von Laeken war sie aufgeworfen und vom ersten Konventsplenum bereits bejaht worden. ${ }^{102}$

Es ist schwierig, genau nachzuweisen, woher die Idee für die schließlich verankerte Unterscheidung zwischen delegierter Rechtsetzung und Durchführungsmaßnahmen stammte und welche Rolle etwa auch der Input aus dem Plenum, Sekretariat und Präsidium spielte. ${ }^{103}$ In den Ausgangspositionen des Europäischen Parlaments findet sich der Vorschlag, Normenkategorien mit verschiedenen Kontrollverfahren einzuführen. Allerdings wird hier noch nicht der Terminus der delegierten Gesetzgebung verwendet und die Vorstellungen von den Kontrollverfahren erschienen noch etwas diffus. ${ }^{104}$ Eine der wenigen Analysen des Zustandekommens dieser Vertragsteile betont zwei Quellen für die letztlich gefundene Formulierung: Die eine Quelle war demnach die Expertenanhörung, und hier besonders die Ausführungen von Koen Lenaerts ${ }^{105}$; die andere Quelle war demnach der Vertreter der Kommission in der Arbeitsgruppe. Dieser habe in einem Arbeitspapier zum einen das Argument favorisiert, dass Gesetzgebung inhaltlich (und nicht nur nach dem Verfahren) zu bestimmen sei, sodass Legislativakte von allgemeiner Tragweite die wesentlichen Elemente eines Regelungsfeldes festlegen. Zum anderen schlug er mit Blick auf den nicht-legislativen Bereich eine Trennung zwischen delegierter Rechtsetzung und rein exekutiven Aufgaben vor. Bekannt ist, dass die Willensbildung in der Arbeitsgruppe wenig kontrovers war und das Schlussdokument breite Unterstützung, auch durch die Vertreter des Europäischen Parlaments, genoss. ${ }^{106}$ Obwohl das Thema in früheren Plenardebatten auch Kontroversen erzeugt hatte, gelang es dem Präsidium schließlich, die Vorschläge der Arbeitsgruppe in konkrete Empfehlungen umzusetzen, die im abschließenden Plenum breite Unterstützung erhielten. ${ }^{107}$

Hier greifen institutionentheoretische Erklärungen. Weil Kommission und Europäisches Parlament Zugang hatten und der Interaktionsmodus jedenfalls auch der des Argumentierens

102 Europäischer Konvent: Aufzeichnung über die Plenartagung - 23./24. Mai 2002, Brüssel, CONV 60/02, S. 5-9; Norman: The Accidental Constitution, 2005, S. 26; Liisberg: The EU Constitutional Treaty and its distinction, 2006.

103 Siehe allgmein auch Norman: The Accidental Constitution, 2005; Tsebelis/Proksch: The Art of Political Manipulation in the European Convention, 2007. Es werden in der Literatur verschiedenste Quellen für die Grundidee der Einführung der delegierten Gesetzgebung im Besonderen und die Idee der Normenhierarchie im Allgemeinen angeführt, so bereits der Spinelli-Entwurf für eine europäische Verfassung von 1984, Konzepte der italienischen Regierung im Kontext der Maastrichter Regierungskonferenz, Konzepte aus dem Ausschuss für konstitutionelle Fragen des Europäischen Parlaments, insbesondere der Bericht Bourlanges und Martin auf der Basis einer Vorlage von Willy Rothley im Vorfeld der Amsterdamer Konferenz. Hofmann nennt (speziell für die Idee der delegierten Rechtsetzung) weitere Quellen in der EuGH-Rechtsprechung. Vgl. Europäischer Konvent: Die Rechtsakte: das derzeitige System, CONV 162/02, Fn. 17; Europäisches Parlament: Bericht über die institutionellen Auswirkungen der Zustimmung des Europäischen Parlaments zur Benennung des Präsidenten der Kommission und über die Unabhängigkeit der Mitglieder des Kollegiums, Institutioneller Ausschuss, Berichterstatter: Elmar Brok, A4-0488/98; Liisberg: The EU Constitutional Treaty and its distinction, 2006, hier S. 10; Hofmann: Legislation, Delegation and Implementation, 2009, S. 495, Fn. 61.

104 Europäisches Parlament: Bericht über die Typologie der Rechtsakte und die Hierarchie der Normen in der Europäischen Union, Ausschuss für konstitutionelle Fragen, Berichterstatter: Jean-Louis Bourlanges, A5 0425/2002, S. 12-13.

105 Koen Lenaerts ist Richter am EuGH und hat sich auch wissenschaftlich mit der Komitologie befasst. In der Anhörung behandelte er vor allem die Unterscheidung zwischen Gesetzgebung und delegierter Rechtsetzung. Liisberg: The EU Constitutional Treaty and its distinction, 2006, S. 13-15.

106 Ebenda, S. 15; Maurer: Deliberation und Bargaining im Konvent, 2006, S. 16.

107 Norman: The Accidental Constitution, 2005; Liisberg: The EU Constitutional Treaty and its distinction, 2006, S. 18. 
und nicht nur der des Verhandelns war ${ }^{108}$ gelang es der Kommission, die Unterscheidung zwischen zwei Normenkategorien einzubringen. Von diesen beiden Normenkategorien sollte nur noch die eine unter Komitologieverfahren zustande kommen - sicherlich auch, weil damit viele langjährige Forderungen des Parlaments erfüllt wurden. Eine weitere Rolle spielte, dass hier tieferliegende Verfassungsvorstellungen zum Tragen kamen, die verschiedene nationale Verfassungstraditionen (so die deutsche und die italienische) reflektierten. ${ }^{109}$ Förderlich war zudem, dass viele Delegierte sich damit mehr Klarheit und Transparenz hinsichtlich der Rechtsakte erhofften. ${ }^{110}$ Neben rational-choice-institutionalistischen Aspekten (Kommission und Parlament haben besseren Zugang zum Prozess der Vertragsänderung) scheinen hier soziologisch-institutionalistische Ansätze (kulturelle Kompatibilitäten mit nationalen Verfassungstraditionen und Legitimationsverlust der bisherigen Konstellation) also ebenso zur Erhellung beizutragen wie historisch-institutionalistische Ansätze: Die beteiligten Regierungsvertreter konnten offenbar die vollen Konsequenzen dieser Neuerung nicht abschätzen.

Gleichwohl stößt man mit den Institutionentheorien an Grenzen, wenn man der Frage nachgeht, warum die Regierungsvertreter, die bei der Regierungskonferenz 2004 am Ende doch über den Verfassungsvertrag zu beschließen hatten, keinen Anstoß an den Neuerungen in den Artt. I-36 und I-37 des Verfassungsvertrags nahmen. ${ }^{111}$ Genau genommen waren es zunächst Rechtsexperten von Rat, Mitgliedstaaten und Kommission, die eine rechtliche Überprüfung des Verfassungsentwurfs vornahmen und auch die Modalitäten der delegierten Rechtsetzung näher prüften, ohne jedoch Änderungen anzuregen. ${ }^{112}$ Dasselbe gilt für die Regierungskonferenz von Lissabon, wo zwei Jahre nach dem Scheitern des Verfassungsvertrags in der Phase der Ratifikation eine verschlankte und von Verfassungsterminologie befreite Fassung des Verfassungsvertrags als Änderungsvertrag des bestehenden Primärrechts beschlossen wurde, das nun mit Artt. 290 und 291 AEUV den identischen Wortlaut der Artt. I-36 und I-37 des Verfassungsvertrag enthält.

Die Ausgestaltung der Komitologieverordnung von 2011 (siehe oben) kann unter Anwendung des Rational-Choice-Institutionalismus ebenso wenig befriedigend erklärt werden. Mit der Frage, warum das Europäische Parlament, das doch mit dem neuen Art. 291 AEUV mit dem Gesetzgebungsverfahren zur Regelung der Komitologieverfahren beste Bedingungen für die weitere Durchsetzung seiner Interessen erhalten hatte, sich bei der Komitologieverordnung und beim Common Understanding so wenig durchsetzte, befasst sich ein Beitrag von Christiansen und Dobbels. Beide argumentieren, dass es den Abgeordneten des Europäischen Parlaments an Zeit mangelte, um eine Strategie für den Umgang mit den Artt. 290 und 291 AEUV zu entwickeln. Sie hätten Schwierigkeiten gehabt, in den Verhandlungen der Expertise von Rat und Kommission etwas entgegen zu setzen. Auch sei es dem Europäischen Parlament schwergefallen, die verschiedenen mitgliedstaatlichen Positionen im Rat für seine Strategie zu nutzen ${ }^{113}$ - eine Argumentation, die man irgendwo zwischen Rational-Choiceund Soziologischem Institutionalismus einordnen könnte. Allerdings überzeugt die Argumentation von Christiansen und Dobbels dort nicht, wo sie vertreten, dass sich das Parlament

108 Zudem stand die Frage der Durchführungsrechtsakte weder in der Konventsarbeitsgruppe noch nach dem Scheitern des Verfassungsvertrags im zentralen politischen Fokus.

109 Liisberg: The EU Constitutional Treaty and its distinction, 2006, S. 42.

110 Ebenda, S. 11.

111 Héritier et al: Changing Roles of Delegation. A Contest for Power in Comitology, 2013, S. 42.

112 Liisberg: The EU Constitutional Treaty and its distinction, 2006, S. 25.

113 Christiansen/Dobbels: Comitology and delegated acts after Lisbon, 2012, S. 5. 
für Fragen der Komitologie traditionell nicht sehr interessiere. ${ }^{114}$ Das Gegenteil ist der Fall, das Parlament hat der Komitologie über die Jahre geradezu übermäßige Aufmerksamkeit geschenkt. Brandsma und Blom-Hansen gehen der ähnlichen Frage nach, warum es in Folge der weitreichenden Reform durch den Lissaboner Vertrag nicht zu weitergehenden Veränderungen der Komitologie gekommen ist ${ }^{115}$ und halten (in einem allerdings eher dünnen rational-choice-institutionalistischen Erklärungskapitel) die Positionen und prozeduralen Ressourcen der Akteure für erklärungskräftig. ${ }^{116}$

Zwei Punkte erscheinen hier bedenkenswert: Zum einen muss betont werden, dass das Europäische Parlament (mehr noch als andere europäische Institutionen) kein einheitlicher Akteur ist. Vielmehr verfolgen insbesondere verschiedene Ausschüsse ganz unterschiedliche Projekte und Agenden. Die Verweise von Christiansen und Dobbels auf verschiedene „Unfälle“, in denen Fachausschüsse Verfahren vorschlugen, die auf der horizontalen Ebene nicht dem Stand der Diskussion entsprachen, ${ }^{117}$ weisen auf den letztlich eher mit dem Soziologischen Institutionalismus zu erklärenden Umstand hin, dass hier unterschiedliche Logiken (vor allem horizontale konstitutionelle und vertikale fachliche) unterschiedliche Resultate hervorbringen. ${ }^{118}$ Dies war bereits in der Vergangenheit häufig der Fall, als beispielsweise der Ausschuss für konstitutionelle Fragen, der Haushaltsausschuss und der Umweltausschuss eine Vielzahl nicht immer kohärenter Positionen hinsichtlich der Komitologie verfolgten. ${ }^{119}$ Zum anderen kann man dem Parlament (als rationalem Akteur) unterstellen, dass es mit der Einführung der delegierten Rechtsetzung vielleicht auch das Interesse an der Komitologie verloren hat, ${ }^{120}$ da es ja als Gesetzgeber durchsetzen kann, wichtige Materien in der delegierten Rechtsetzung zu platzieren. Ob es in diesen Verfahren dann die nötigen Ressourcen aufbringt, um deren Ausübung auch effektiv zu überwachen, ist eine weitere noch unbeantwortete Frage.

Des Weiteren sind auch die Mitgliedstaaten, wie vom Mehrebenenansatz betont, keine einheitlichen, durchweg rational und strategisch agierenden Akteure. Während die Generalisten aus den nationalen Exekutiven im Konvent Verfahren zugestimmt haben, die - abstrakt betrachtet - aufgrund nationaler Verfassungstraditionen akzeptabel erschienen, fiel den Experten in den Fachministerien erst sehr viel später auf, was die neuen Regelungen für die Verfahren und Resultate in den jeweiligen Politikfeldern bedeuten würden. ${ }^{121}$ Und auch der Rat erkannte nach Einschätzung verschiedener Beobachter erst nach der Ratifikation des Lissaboner Vertrags, dass mit Art. 290 AEUV ein Verfahren geschaffen worden war, in dem es keine Komitologie mehr geben würde, was der Rat als klaren Machtverlust wahrnimmt. ${ }^{122}$ Solche nicht vollständig von mächtigen Akteuren gesteuerten Entwicklungen erklärt uns vor allem der Historische Institutionalismus. Schließlich spielen gerade in der Anwendung der hier behandelten neuen Regelungen jenseits von Machtfragen auch Fragen

114 Ebenda, S. 15.

115 Brandsma/Blom-Hansen: Negotiating the Post-Lisbon Comitology System, 2012, S. 940.

116 Ebenda, S. 954.

117 Vgl. zum Beispiel Christiansen/Dobbels: Comitology and delegated acts after Lisbon, 2012, S. 12.

118 Vgl. auch Brandsma/Blom-Hansen: Negotiating the Post-Lisbon Comitology System, 2012, S. 952.

119 Töller: Komitologie, 2002, S. 250-251.

120 Christiansen/Dobbels: Comitology and delegated acts after Lisbon, 2012, hier S. 15.

121 Für diesen Hinweis danke ich Andreas Maurer.

122 Buchet et al.: Changing Rules of Delegation, 2012, Min. 41:00 und 47:00. 
kultureller Kompatibilität zwischen Institutionen eine wichtige Rolle ${ }^{123}$ - womit wir wiederum bei soziologisch-institutionellen Erklärungsansätzen wären.

\section{Resümee: Es bleiben noch einige Rätsel ungelöst}

Die Komitologie, also die Delegation von Regelungs- und Durchführungskompetenzen an die Kommission und deren Wahrnehmung unter Beteiligung von Ausschüssen, hat sich seit den 1960er Jahren inkrementell entwickelt, ausdifferenziert und in beinahe allen Politikfeldern verbreitet. 1987 unternahm man den Versuch einer Konstitutionalisierung, es folgten Reformen der Verfahren 1999 und 2006, die vor allem dazu dienen sollten, die zunehmenden Forderungen des Europäischen Parlaments (als erstarkter Bestandteil des europäischen Gesetzgebers) zu berücksichtigen. Der Lissaboner Vertrag brachte eine in ihrer Radikalität überraschende Reform, die in ihrem Inhalt auf den Verfassungsentwurf des Konvents und innerhalb des Konvents wesentlich auf die Arbeitsgruppe Vereinfachung zurückgeht. Während aber die Reform auf der Vertragsebene zunächst das Parlament als Gewinner erscheinen ließ, sieht man inzwischen die Kommission als Profiteurin der neuen Rechtslage. In diese Richtung weist auch das Ergebnis der Verfahrensreform unterhalb des Primärrechts, also das Common Understanding zu Art. 290 AEUV und die Komitologieverordnung zur Konkretisierung von Art. 291 AEUV. Zum einen blieb das Komitologiesystem im Wesentlichen bestehen, auch wenn die Verfahrensvielfalt reduziert und der Rekurs zum Rat abgeschafft wurde, zum anderen setzte hier (trotz gleichberechtigter Beteiligung) das Parlament kaum Forderungen durch.

Rational-choice-institutionentheoretische Ansätze, die die Gemeinschaftsinstitutionen Kommission, Parlament und Rat beziehungsweise auch die Mitgliedstaaten als Akteure mit klaren Präferenzen und unterschiedlichen Ressourcen verstehen, können das Zustandekommen der überraschend radikalen Reform ebenso wie die - angesichts des ordentlichen Gesetzgebungsverfahrens - überraschend wenig radikale Reform der Komitologiemodalitäten nur bedingt erklären. Vieles in der europäischen Politik ist vielschichtiger, als rein rationalchoice-orientierte Ansätze es vermuten lassen. Mit einer erweiterten Perspektive, die mit dem Historischen und dem Soziologischen Institutionalismus auch kulturelle Faktoren und nichtintendierte Folgen mit einschließt, kann man einiges mehr erklären. Es bleiben aber doch noch Rätsel für die zukünftige Forschung übrig. Das größte Rätsel bleibt, warum die revolutionäre Einführung der delegierten Rechtsetzung unter Abschaffung der Komitologie zwei Regierungskonferenzen überstanden hat, ohne von den Mitgliedstaaten wieder aus dem Vertrag gestrichen zu werden.

Die Effekte dieser Reform sind noch nicht genau abzusehen. Möglich ist, dass der Gesetzgeber nun großzügiger delegiert (weil er ja ein Rückholrecht hat und daher nichts riskiert) und dies der Entscheidungseffizienz und der Transparenz der Union zuträglich ist. Möglich ist aber auch, dass neue Konflikte zwischen Parlament und Rat über die rechtlich unklare Unterscheidung zwischen Artt. 290 und 291 AEUV oder über die genaue Ausgestaltung des Verfahrens nach Art. 290 AEUV das Tagesgeschäft bestimmen werden. Die meisten Beobachter rechnen damit, dass hierüber bald der EuGH zu entscheiden hat.

123 So argumentiert Buchet, jenseits aller Rhetorik des supranationalen Schulterschlusses zwischen Kommission und Parlament seien sich Beamte von Kommission und Rat kulturell einfach vertrauter. Vgl. Buchet et al.: Changing Rules of Delegation, 2012, Min. 31:30. 